Supplement of

\title{
A long-term estimation of biogenic volatile organic compound (BVOC) emission in China from 2001-2016: the roles of land cover change and climate variability
}

Hui Wang et al.

Correspondence to: Qizhong Wu (wqizhong@bnu.edu.cn) and Lanning Wang (wangln@bnu.edu.cn)

The copyright of individual parts of the supplement might differ from the article licence. 
Table S1. Look-up table for mapping the IGBP legend to eight main vegetations categories.

\begin{tabular}{|c|c|c|c|}
\hline Name & Value & Description & $\begin{array}{l}\text { Main Category } \\
\text { Percentage }\end{array}$ \\
\hline $\begin{array}{l}\text { Needleleaf } \\
\text { Evergreen Forest }\end{array}$ & 1 & $\begin{array}{l}\text { Dominated by evergreen conifer } \\
\text { trees (canopy }>2 \mathrm{~m} \text { ). }\end{array}$ & $100 \%$ NET \\
\hline $\begin{array}{l}\text { Broadleaf } \\
\text { Evergreen Forest }\end{array}$ & 2 & $\begin{array}{l}\text { Dominated by evergreen broadleaf } \\
\text { and palmate trees (canopy }>2 \mathrm{~m} \text { ). }\end{array}$ & $100 \% \mathrm{BET}$ \\
\hline $\begin{array}{l}\text { Needleleaf } \\
\text { Deciduous Forest }\end{array}$ & 3 & $\begin{array}{l}\text { Dominated by deciduous needleleaf } \\
\text { (larch) trees (canopy }>2 \mathrm{~m} \text { ). }\end{array}$ & $100 \%$ NDT \\
\hline $\begin{array}{l}\text { Broadleaf } \\
\text { Deciduous Forest }\end{array}$ & 4 & $\begin{array}{l}\text { Dominated by deciduous broadleaf } \\
\text { trees (canopy }>2 \mathrm{~m} \text { ). }\end{array}$ & $100 \% \mathrm{BDT}$ \\
\hline Mixed Forests & 5 & $\begin{array}{l}\text { Dominated by neither deciduous nor } \\
\text { evergreen }(40-60 \% \text { of each) tree } \\
\text { type (canopy }>2 \mathrm{~m}) \text {. }\end{array}$ & $100 \%$ Mixed Forests \\
\hline Closed Shrublands & 6 & $\begin{array}{l}\text { Dominated by woody perennials ( } 1- \\
2 \mathrm{~m} \text { height) }>60 \% \text { cover. }\end{array}$ & $100 \%$ Shrub \\
\hline Open Shrublands & 7 & $\begin{array}{l}\text { Dominated by woody perennials ( } 1- \\
2 \mathrm{~m} \text { height) } 10-60 \% \text { cover. }\end{array}$ & $\begin{array}{l}60 \% \text { Shrub } \\
40 \% \text { Grass }\end{array}$ \\
\hline Woody Savannas & 8 & Tree cover $30-60 \%$ (canopy $>2 \mathrm{~m}$ ). & $\begin{array}{l}60 \% \text { Mixed Forest } \\
20 \% \text { Shrub } \\
20 \% \text { Grass }\end{array}$ \\
\hline Savannas & 9 & Tree cover $10-30 \%$ (canopy $>2 \mathrm{~m}$ ). & $\begin{array}{l}30 \% \text { Mixed Forest } \\
35 \% \text { Shrub } \\
35 \% \text { Grass }\end{array}$ \\
\hline Grasslands & 10 & $\begin{array}{l}\text { Dominated by herbaceous annuals } \\
(<2 \mathrm{~m}) \text {. }\end{array}$ & $100 \%$ Grass \\
\hline
\end{tabular}




\begin{tabular}{|c|c|c|c|}
\hline $\begin{array}{l}\text { Permanent } \\
\text { Wetlands }\end{array}$ & 11 & $\begin{array}{l}\text { Permanently inundated lands with } \\
30-60 \% \text { water cover and }>10 \% \\
\text { vegetated cover. }\end{array}$ & $40 \%$ Grass \\
\hline Croplands & 12 & $\begin{array}{l}\text { At least } 60 \% \text { of area is cultivated } \\
\text { cropland. }\end{array}$ & $100 \%$ Crop \\
\hline $\begin{array}{l}\text { Urban and Built-up } \\
\text { Lands }\end{array}$ & 13 & $\begin{array}{l}\text { At least } 30 \% \text { impervious surface } \\
\text { area including building materials, } \\
\text { asphalt, and vehicles. }\end{array}$ & None \\
\hline $\begin{array}{l}\text { Cropland/Natural } \\
\text { Vegetation Mosaics }\end{array}$ & 14 & $\begin{array}{l}\text { Mosaics of small-scale cultivation } \\
40-60 \% \text { with natural tree, shrub, or } \\
\text { herbaceous vegetation. }\end{array}$ & $\begin{array}{l}60 \% \text { Crop } \\
20 \% \text { Shrub } \\
20 \% \text { Grass }\end{array}$ \\
\hline $\begin{array}{l}\text { Permanent Snow } \\
\text { and Ice }\end{array}$ & 15 & $\begin{array}{l}\text { At least } 60 \% \text { of area is covered by } \\
\text { snow and ice for at least } 10 \text { months } \\
\text { of the year. }\end{array}$ & None \\
\hline Barren & 16 & $\begin{array}{l}\text { At least } 60 \% \text { of area is non- } \\
\text { vegetated barren (sand, rock, soil) } \\
\text { areas with less than } 10 \% \text { vegetation. }\end{array}$ & None \\
\hline
\end{tabular}

Table S2. The climatic criteria for mapping main vegetation categories to CLM PFTs.

\begin{tabular}{l|l|l}
\hline Main Category & Mapping Condition & CLM PFT \\
\hline \multirow{2}{*}{ NET } & $\mathrm{T}_{\mathrm{c}}>-19^{\circ} \mathrm{C}$ and GDD $>1200$ & $100 \%$ NET Temperate \\
\cline { 2 - 3 } & $\mathrm{T}_{\mathrm{c}} \leq-19^{\circ} \mathrm{C}$ or GDD $\leq 1200$ & $100 \%$ NET Boreal \\
\hline \multirow{2}{*}{ BET } & $\mathrm{T}_{\mathrm{c}}>15.5^{\circ} \mathrm{C}$ & $100 \%$ BET Tropical \\
\cline { 2 - 3 } & $\mathrm{T}_{\mathrm{c}} \leq 15.5^{\circ} \mathrm{C}$ & $100 \%$ BET Temperate \\
\hline \multirow{2}{*}{ BDT } & $\mathrm{None}^{\circ}$ & $100 \%$ NDT \\
\hline & $\mathrm{T}_{\mathrm{c}}>15.5^{\circ} \mathrm{C}$ & $100 \%$ BDT Tropical \\
\cline { 2 - 3 } & $-15.5{ }^{\circ} \mathrm{C}<\mathrm{T}_{\mathrm{c}} \leq 15.5 \quad{ }^{\circ} \mathrm{C}$ or & $100 \%$ BDT Temperate \\
\hline \multirow{2}{*}{ Mixed Forest } & $\mathrm{GDD}>1200$ & $100 \%$ BDT Boreal \\
\cline { 2 - 3 } & $\mathrm{T}_{\mathrm{c}} \leq-15.5^{\circ} \mathrm{C}$ or GDD $\leq 1200$ & $50 \%$ BET Tropical \\
\hline
\end{tabular}




\begin{tabular}{|c|c|c|}
\hline & & $50 \%$ BDT Tropical \\
\hline & \multirow{3}{*}{$\begin{array}{l}-15.5 \quad{ }^{\circ} \mathrm{C}<\mathrm{T}_{\mathrm{c}} \leq 15.5 \quad{ }^{\circ} \mathrm{C} \text { and } \\
\mathrm{GDD}>1200\end{array}$} & $33.33 \%$ NET Temperate \\
\hline & & $33.33 \%$ BET Temperate \\
\hline & & 33.33\% BDT Temperate \\
\hline & \multirow[t]{3}{*}{$\mathrm{T}_{\mathrm{c}} \leq-15.5^{\circ} \mathrm{C}$ or $\mathrm{GDD} \leq 1200$} & $33.33 \%$ NDT \\
\hline & & 33.33\% NET Boreal \\
\hline & & $33.33 \%$ BDT Boreal \\
\hline \multirow[t]{2}{*}{ Shrub } & $\mathrm{T}_{\mathrm{c}}>-19^{\circ} \mathrm{C}$ and GDD $>1200$ & $100 \%$ BDS Temperate \\
\hline & $\mathrm{T}_{\mathrm{c}} \leq-19^{\circ} \mathrm{C}$ or GDD $\leq 1200$ & $100 \%$ BDS Boreal \\
\hline \multirow[t]{3}{*}{ Grass } & $\mathrm{GDD}<1000$ & 100\% C3 Arctic \\
\hline & $\begin{array}{l}\mathrm{GDD}>1000 \text { and }(\mathrm{Tc} \leq \\
\left.22^{\circ} \mathrm{C} \text { or Pmon } \leq 25 \mathrm{~mm}\right)\end{array}$ & $100 \% \mathrm{C} 3$ \\
\hline & $\begin{array}{l}\mathrm{GDD}>1000 \text { and } \mathrm{Tc}>22^{\circ} \mathrm{C} \\
\text { and Pmon }>25 \mathrm{~mm}\end{array}$ & $100 \% \mathrm{C} 4$ \\
\hline Crop & None & $100 \%$ Crop \\
\hline
\end{tabular}

Table S3. MEGAN2.1 biogenic emission classes and emission factors (ug m-2 h-1) for each of the plant functional types (Guenther et al., 2012). $\mathrm{EF}_{1} \sim \mathrm{EF}_{15}$ represents different PFTs, and they are needle evergreen temperate trees $\left(\mathrm{EF}_{1}\right)$, needle evergreen boreal trees $\left(\mathrm{EF}_{2}\right)$, needle deciduous boreal trees $\left(\mathrm{EF}_{3}\right)$, broadleaf evergreen tropical tree $\left(\mathrm{EF}_{4}\right)$, broadleaf evergreen temperate tree $\left(\mathrm{EF}_{5}\right)$, broadleaf deciduous tropical tree $\left(\mathrm{EF}_{6}\right)$, broadleaf deciduous temperate tree $\left(\mathrm{EF}_{7}\right)$, broadleaf deciduous boreal tree $\left(\mathrm{EF}_{8}\right)$, broadleaf evergreen temperate shrub $\left(\mathrm{EF}_{9}\right)$, broadleaf deciduous 
temperate shrub $\left(\mathrm{EF}_{10}\right)$, broadleaf deciduous boreal shrub $\left(\mathrm{EF}_{11}\right)$, arctic $\mathrm{C} 3$ grass $\left(\mathrm{EF}_{12}\right)$, cool $\mathrm{C} 3$ grass $\left(\mathrm{EF}_{13}\right)$, warm $\mathrm{C} 4$ grass $\left(\mathrm{EF}_{14}\right)$ and crop $\left(\mathrm{EF}_{15}\right)$.

\begin{tabular}{|c|c|c|c|c|c|c|c|c|c|c|c|c|c|c|c|}
\hline Compound Class & $\mathrm{EF}_{1}$ & $\mathrm{EF}_{2}$ & $\mathrm{EF}_{3}$ & $\mathrm{EF}_{4}$ & $\mathrm{EF}_{5}$ & $\mathrm{EF}_{6}$ & $\mathrm{EF}_{7}$ & $\mathrm{EF}_{8}$ & $\mathrm{EF}_{9}$ & $\mathrm{EF}_{10}$ & $\mathrm{EF}_{11}$ & $\mathrm{EF}_{12}$ & $\mathrm{EF}_{13}$ & $\mathrm{EF}_{14}$ & $\mathrm{EF}_{15}$ \\
\hline Isoprene & 600 & 3000 & 1 & 7000 & 10000 & 7000 & 10000 & 11000 & 2000 & 4000 & 4000 & 1600 & 800 & 200 & 1 \\
\hline Myrcene & 70 & 70 & 60 & 80 & 30 & 80 & 30 & 30 & 30 & 50 & 30 & 0.3 & 0.3 & 0.3 & 0.3 \\
\hline Sabinene & 70 & 70 & 40 & 80 & 50 & 80 & 50 & 50 & 50 & 70 & 50 & 0.7 & 0.7 & 0.7 & 0.7 \\
\hline Limonene & 100 & 100 & 130 & 80 & 80 & 80 & 80 & 80 & 60 & 100 & 60 & 0.7 & 0.7 & 0.7 & 0.7 \\
\hline 3-Carene & 160 & 160 & 80 & 40 & 30 & 40 & 30 & 30 & 30 & 100 & 30 & 0.3 & 0.3 & 0.3 & 0.3 \\
\hline$t$ - $\beta$-Ocimene & 70 & 70 & 60 & 150 & 120 & 150 & 120 & 120 & 90 & 150 & 90 & 2 & 2 & 2 & 2 \\
\hline$\beta$-Pinene & 300 & 300 & 200 & 120 & 130 & 120 & 130 & 130 & 100 & 150 & 100 & 1.5 & 1.5 & 1.5 & 1.5 \\
\hline$\alpha$-Pinene & 500 & 500 & 510 & 600 & 400 & 600 & 400 & 400 & 200 & 300 & 200 & 2 & 2 & 2 & 2 \\
\hline Other Monoterpenes & 180 & 180 & 170 & 150 & 150 & 150 & 150 & 150 & 110 & 200 & 110 & 5 & 5 & 5 & 5 \\
\hline$\alpha$-Farnesene & 40 & 40 & 40 & 60 & 40 & 60 & 40 & 40 & 40 & 40 & 40 & 3 & 3 & 3 & 4 \\
\hline$\beta$-Caryophyllene & 80 & 80 & 80 & 60 & 40 & 60 & 40 & 40 & 50 & 50 & 50 & 1 & 1 & 1 & 4 \\
\hline Other Sesquiterpenes & 120 & 120 & 120 & 120 & 100 & 120 & 100 & 100 & 100 & 100 & 100 & 2 & 2 & 2 & 2 \\
\hline $232-\mathrm{MBO}$ & 700 & 60 & 0.01 & 0.01 & 0.01 & 0.01 & 0.01 & 2 & 0.01 & 0.01 & 0.01 & 0.01 & 0.01 & 0.01 & 0.01 \\
\hline Methanol & 900 & 900 & 900 & 500 & 900 & 500 & 900 & 900 & 900 & 900 & 900 & 500 & 500 & 500 & 900 \\
\hline Acetone & 240 & 240 & 240 & 240 & 240 & 240 & 240 & 240 & 240 & 240 & 240 & 80 & 80 & 80 & 80 \\
\hline $\mathrm{CO}$ & 600 & 600 & 600 & 600 & 600 & 600 & 600 & 600 & 600 & 600 & 600 & 600 & 600 & 600 & 600 \\
\hline Bidirectional VOC & 500 & 500 & 500 & 500 & 500 & 500 & 500 & 500 & 500 & 500 & 500 & 80 & 80 & 80 & 80 \\
\hline Stress VOC & 300 & 300 & 300 & 300 & 300 & 300 & 300 & 300 & 300 & 300 & 300 & 300 & 300 & 300 & 300 \\
\hline Other VOC & 140 & 140 & 140 & 140 & 140 & 140 & 140 & 140 & 140 & 140 & 140 & 140 & 140 & 140 & 140 \\
\hline
\end{tabular}

Table S4. The physical schemes for the WRF simulation.

\begin{tabular}{c|c}
\hline Physical mechanism & Scheme \\
\hline Microphysics & WSM 3-class simple ice scheme \\
\hline Long-wave radiation & RRTM scheme \\
\hline Short-wave radiation & Duhbia scheme \\
\hline Land Surface & Noah Land Surface Model \\
\hline PBL Sche & YSU scheme \\
\hline Cumulus parameter & Kain-Fritsch (new Eta) scheme
\end{tabular}



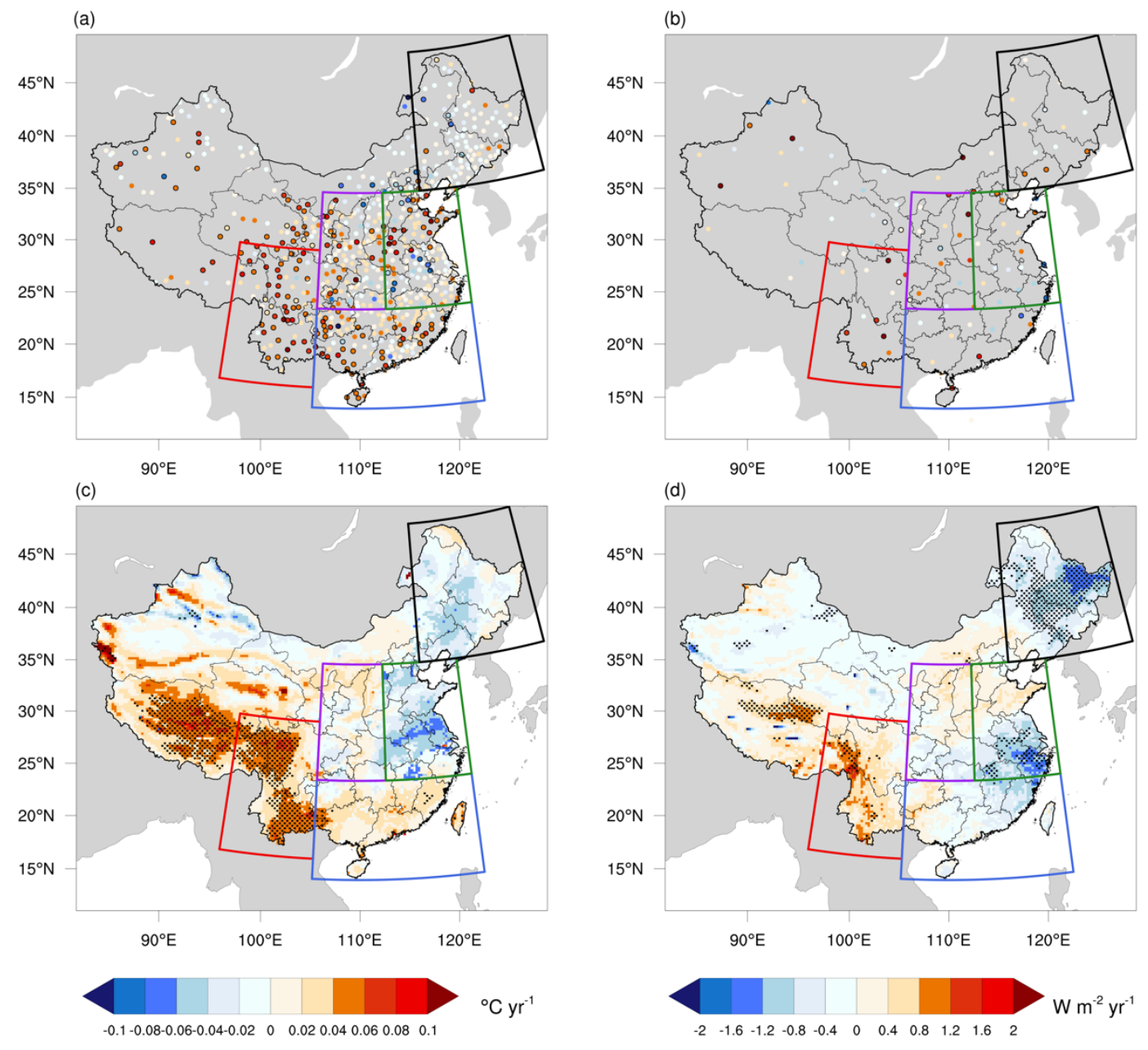

Figure S1. The trend of growing season averaged 2-meter temperature (T2) and downward shortwave radiation (DSW). (a) and (b) are for in-situ T2 and DSW, respectively, and the sites with statistically significant trend are marked by black circles. (c) and (d) are for the WRF simulated T2 and DSW, respectively, and the regions with statistically significant trend are illustrated by shadow. The boxes represent the sub-regions chosen for the regional validation of meteorology, and these regions include Northeastern (black), Central (purple), Eastern (green), Southeastern (red) and Southern (blue) China. 

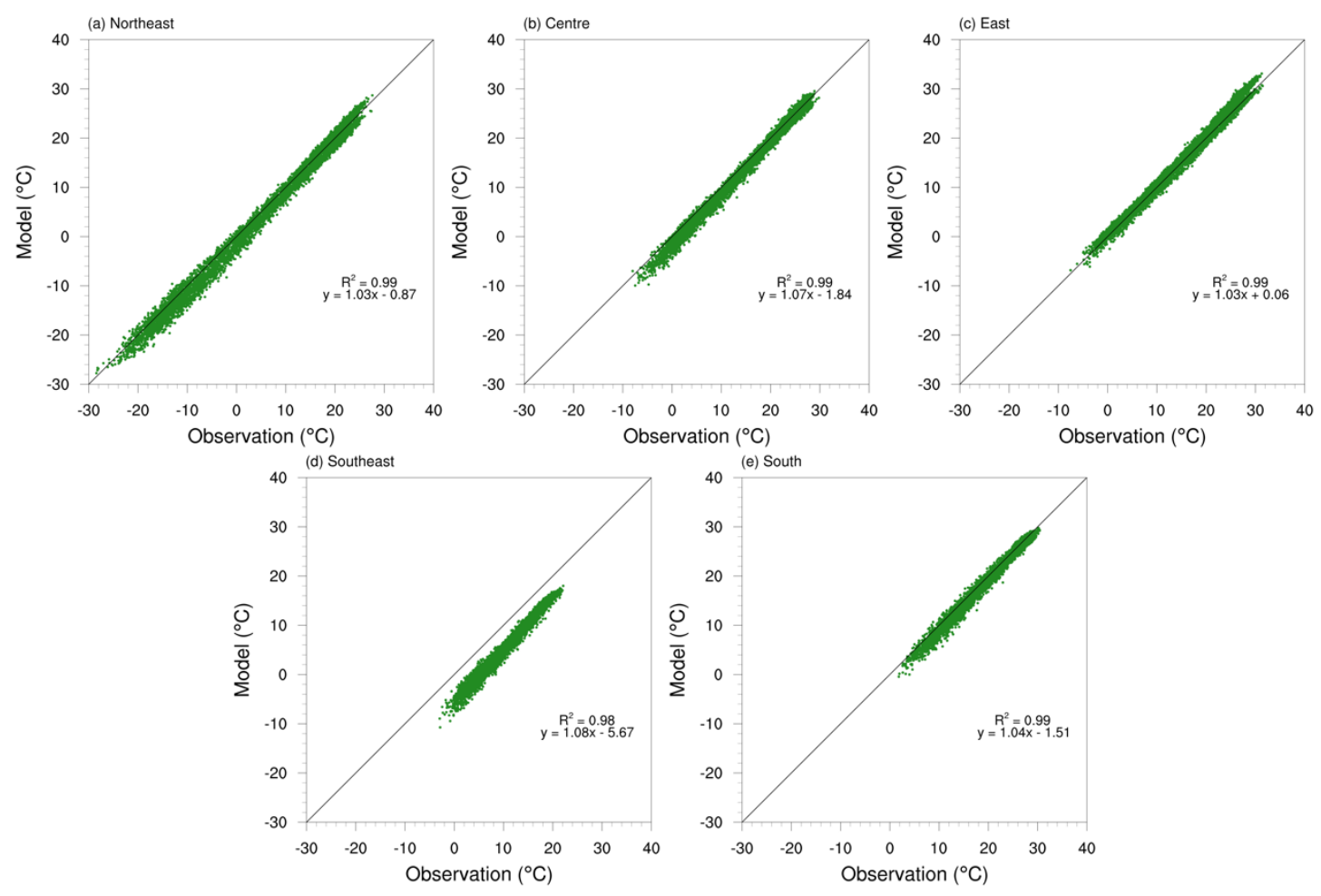

Figure S2. Validations of the daily 2-meter temperature in different regions.

Table S5. The statistic parameters for the validation of the region averaged 2-meter temperature in different regions.

MB, ME and RMSE are shorts for mean bias, mean error and root mean square error, respectively.

\begin{tabular}{l|l|l|l|l}
\hline & $\mathrm{R}$ & $\mathrm{MB}\left({ }^{\circ} \mathrm{C}\right)$ & $\mathrm{ME}\left({ }^{\circ} \mathrm{C}\right)$ & $\mathrm{RMSE}\left({ }^{\circ} \mathrm{C}\right)$ \\
\hline Northeast & 0.99 & -0.71 & 1.31 & 1.60 \\
\hline Centre & 0.99 & -0.89 & 1.12 & 1.42 \\
\hline East & 0.99 & 0.50 & 0.95 & 1.13 \\
\hline Southeast & 0.99 & -4.70 & 4.70 & 4.80 \\
\hline South & 0.99 & -0.71 & 0.88 & 1.13 \\
\hline
\end{tabular}



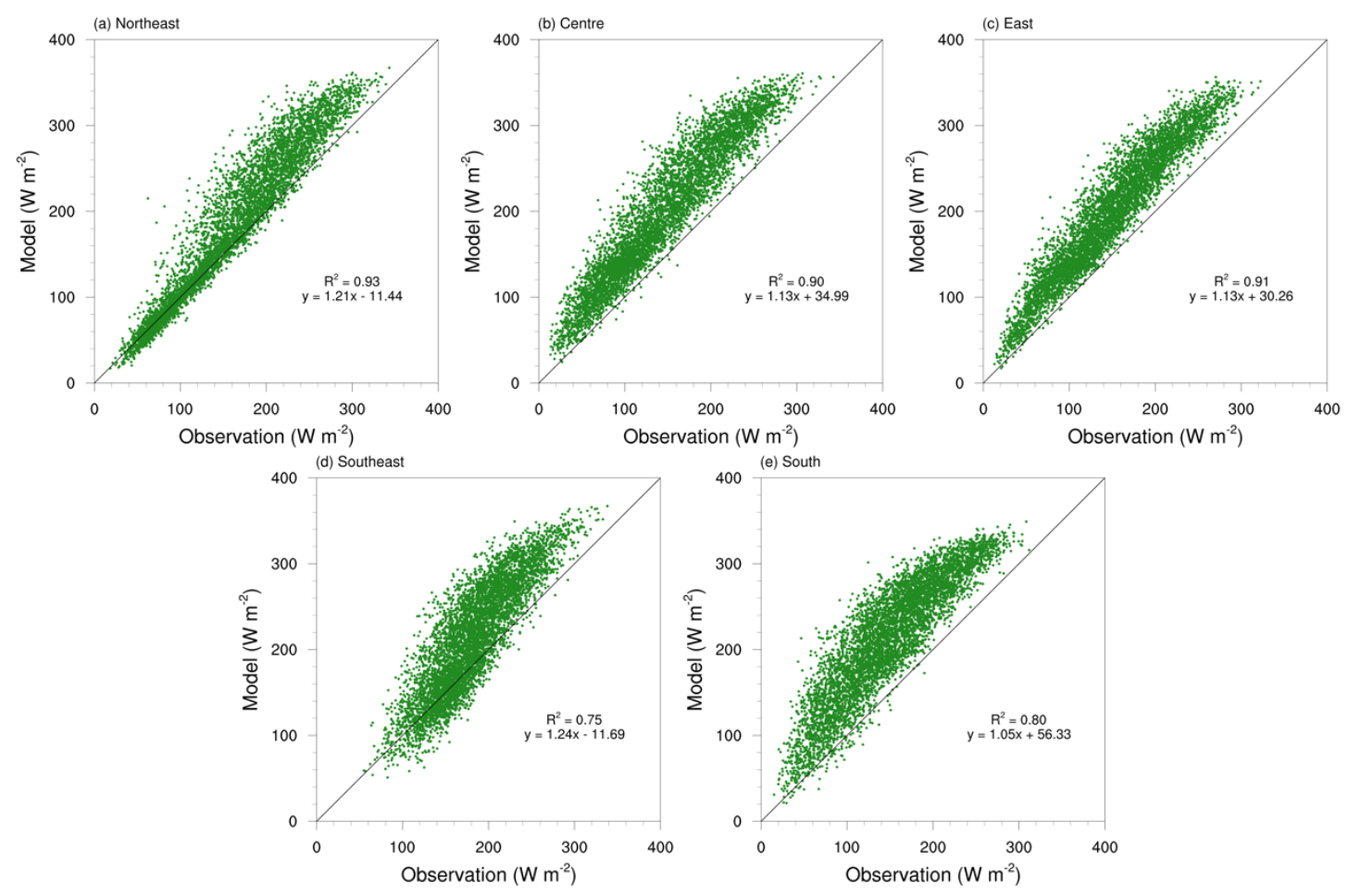

Figure S3. Validations of the daily downward shortwave radiation in different regions.

Table S6. The statistic parameters for the validation of the region averaged downward shortwave radiation in different regions. MB, ME and RMSE are shorts for mean bias, mean error and root mean square error, respectively.

\begin{tabular}{l|l|l|l|l}
\hline & $\mathrm{R}$ & $\mathrm{MB}\left(\mathrm{W} \mathrm{m}^{-2}\right)$ & $\mathrm{ME}\left(\mathrm{W} \mathrm{m}^{-2}\right)$ & $\mathrm{RMSE}\left(\mathrm{W} \mathrm{m}^{-2}\right)$ \\
\hline Northeast & 0.96 & 21.93 & 24.94 & 35.37 \\
\hline Centre & 0.95 & 53.60 & 53.66 & 59.66 \\
\hline East & 0.95 & 48.99 & 49.01 & 54.34 \\
\hline Southeast & 0.87 & 31.91 & 37.61 & 46.38 \\
\hline South & 0.90 & 64.26 & 64.51 & 71.13 \\
\hline
\end{tabular}

\section{Reference}

Guenther, A. B., Jiang, X., Heald, C. L., Sakulyanontvittaya, T., Duhl, T., Emmons, L. K., and Wang, X.: The Model of Emissions of Gases and Aerosols from Nature version 2.1 (MEGAN2.1): an extended and updated framework for modeling biogenic emissions, Geoscientific Model Development, 5, 1471-1492, 10.5194/gmd-5-1471-2012, 2012. 
